\title{
REVIEW \\ Rethinking phenotypic plasticity and its consequences for individuals, populations and species
}

\begin{abstract}
A Forsman
Much research has been devoted to identify the conditions under which selection favours flexible individuals or genotypes that are able to modify their growth, development and behaviour in response to environmental cues, to unravel the mechanisms of plasticity and to explore its influence on patterns of diversity among individuals, populations and species. The consequences of developmental plasticity and phenotypic flexibility for the performance and ecological success of populations and species have attracted a comparatively limited but currently growing interest. Here, I re-emphasize that an increased understanding of the roles of plasticity in these contexts requires a 'whole organism' (rather than 'single trait') approach, taking into consideration that organisms are integrated complex phenotypes. I further argue that plasticity and genetic polymorphism should be analysed and discussed within a common framework. I summarize predictions from theory on how phenotypic variation stemming from developmental plasticity and phenotypic flexibility may affect different aspects of population-level performance. I argue that it is important to distinguish between effects associated with greater interindividual phenotypic variation resulting from plasticity, and effects mediated by variation among individuals in the capacity to express plasticity and flexibility as such. Finally, I claim that rigorous testing of predictions requires methods that allow for quantifying and comparing whole organism plasticity, as well as the ability to experimentally manipulate the level of and capacity for developmental plasticity and phenotypic flexibility independent of genetic variation.

Heredity (2015) 115, 276-284; doi:10.1038/hdy.2014.92; published online 8 October 2014
\end{abstract}

\section{INTRODUCTION}

The focus of this review is 'whole organism' (rather than 'single trait') plasticity, how it may affect the ecological success of individuals, populations and species, and how to rigorously test predictions from theory regarding its consequences. Knowledge of the causes, mechanistic underpinnings and consequences of phenotypic plasticity and the capacity of a genotype to produce different phenotypes in response to environmental variation is crucial for a better understanding of the evolution and maintenance of biodiversity (Bradshaw, 1965; Sultan, 2000; Agrawal, 2001; Pigliucci, 2001; Meyers and Bull, 2002; Bolnick et al., 2003; Booth and Grime, 2003; Sultan, 2004; Miner et al., 2005; Pigliucci, 2005; Gray and McKinnon, 2007; Whitlock et al., 2007; Forsman et al., 2008; Naeem et al., 2009; Whitman and Agrawal, 2009; Pfennig et al., 2010; Reed et al., 2010; te Beest et al., 2011; Violle et al., 2012; Wund, 2012; Snell-Rood, 2013). From having been considered primarily as nuisance in evolutionary biology, plasticity research has grown tremendously (Scheiner and DeWitt, 2004), from $<10$ papers published per year before 1983 to nearly 1300 papers in 2013 (Figure 1). To what extent has this growing attention, appreciation of the potential importance of plasticity and increased scientific output been accompanied by a similar increase in our understanding and knowledge of the causes and consequences of plasticity?

Much research has been devoted to unravel how plasticity may influence and contribute to diversity among individuals, populations and species. Key questions in this area include, but are not limited to:
Does plasticity promote or hinder evolution and speciation? Why does plasticity vary among populations and species? Is plasticity adaptive? What are the costs of plasticity? Do genes typically act as 'leaders' or 'followers' in evolution? What is the relative contribution of plasticity versus evolution (via modifications of allele frequencies) to population differentiation? What are the ecological conditions that promote the evolution and maintenance of plasticity? Some issues, such as the genetic underpinnings of plasticity and gene-by-environment interactions, have been settled or superseded by new questions and approaches (Pigliucci, 2005). Plasticity can be considered at the level of genes, individuals, and populations (see, for example, Carroll and Corneli, 1995; Colbourne et al., 1997; Pigliucci et al., 2003; Nussey et al., 2007; Chun, 2011; Brommer, 2013; Araya-Ajoy and Dingemanse, 2014), but there are also studies based on comparisons of plasticity among species (see, for example, Thaler and Karban, 1997; Pigliucci et al., 1999; Pollard et al., 2001; Davidson et al., 2011). Here, I focus primarily on the consequences for individuals and populations of whole organism developmental plasticity and phenotypic flexibility. An enhanced knowledge of the population-level consequences of interindividual genetic and phenotypic variation may increase our ability to understand the ecological dynamics of natural populations, and ultimately to develop more informed management plans for protection and restoration of threatened species and biodiversity (Kendall and Fox, 2002; Frankham et al., 2010; Wennersten and Forsman, 2012). 


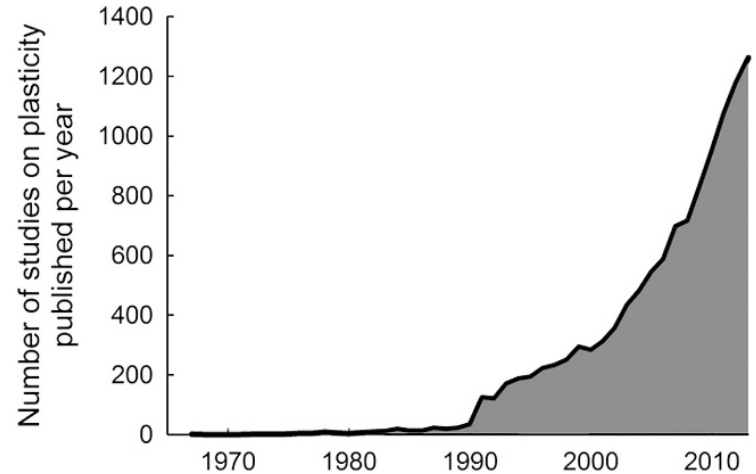

Figure 1 Trends in research output on plasticity. Figure shows number of publications per year up to December 2013. Figure is based on data extracted from a topic search for 'phenotypic plasticity OR 'developmental plasticity' OR 'phenotypic flexibility' OR 'behavio*ral plasticity' conducted on 21 March 2014 from ISI Web of Science. The literature search yielded 11822 papers published between 1967 and December 2013. More than 1000 papers are review articles.

\section{FROM CAUSES TO POPULATION-LEVEL CONSEQUENCES OF PLASTICITY}

In recent years, there has been an increased interest in the questions of how interindividual variation and plasticity influence the performance and ecological success of individuals, populations and species (Sultan, 2000; Agrawal, 2001; Pigliucci, 2001; Bolnick et al., 2003; Miner et al., 2005; Forsman et al., 2008; Hughes et al., 2008; Chevin et al., 2010; Pfennig et al., 2010; Violle et al., 2012; Wennersten and Forsman, 2012). Overall, theoretical models agree that phenotypically and genetically more variable populations are predicted to be associated with broader niches, reduced intraspecific competition, increased productivity and population growth rate, decreased vulnerability to environmental changes, dampened fluctuations in population size, increased establishment success, increased invasiveness, larger distribution ranges, decreased extinction risk and increased rate of speciation as compared with less variable populations (reviewed by Forsman et al., 2008; Wennersten and Forsman, 2012 and references therein). Wennersten and Forsman (2012) systematically evaluate whether the consequences of interindividual variation are likely to be similar or different depending on the nature of the source of variation. Despite some exceptions with regard to temporal and spatial scales, the population-level consequences listed above are, at first glance, largely similar regardless of whether the variation stems from genetic polymorphism (allelic variation at coding loci), plasticity or randomized phenotype switching (see Table 1 in Wennersten and Forsman, 2012).

The overall agreement with regard to consequences is in part illusory, because the mechanisms that mediate the consequences of plasticity versus polymorphism are sometimes different (for a discussion and examples see Wennersten and Forsman, 2012). Furthermore, predictions are sometimes similar because models on the consequences of plasticity build in part on the assumption that there are heritable differences among genotypes in norms of reaction (how the phenotypic expression of genotypes change along an environmental gradient), in which case the distinction between genetic polymorphism and plasticity is debatable (West-Eberhard, 2003; Leimar, 2009; Shuster, 2010; Wennersten and Forsman, 2012). The consequences of plasticity for the ecological success of populations and individuals may also differ depending on whether one is concerned with variation among individuals in the capacity to express plasticity, or with
Table 1 List of plasticity-related terms and concepts, arranged in the order they appear in the text

\begin{tabular}{l}
\hline Phenotypic plasticity \\
\hline Gene by environment interaction \\
Developmental plasticity \\
Phenotypic flexibility \\
Norms of reaction \\
Induced responses \\
Intraindividual plasticity \\
Induced differentiation \\
Active plasticity \\
Passive plasticity \\
Inducible defences \\
Phenotypic flexibility \\
Reaction norms \\
Maternal effects \\
Cross generational plasticity \\
Reversible changes within individuals \\
Labile traits \\
Behavioural plasticity \\
Activational plasticity \\
Irreversible developmental plasticity \\
Window of sensitivity \\
Multivariate trait plasticity \\
\hline The list is nonexhaustive, and additional terms and concepts can be found in the plasticity
\end{tabular}

The list is nonexhaustive, and additional terms and concepts can be found in the plasticity literature.

interindividual phenotypic variation resulting from induced responses (which could also arise in the absence of genetic variation for plasticity if individuals utilize different microhabitats).

Developing, summarizing and comparing theoretical models and their predictions (see, for instance, Table 1 in Wennersten and Forsman, 2012) are necessary and important tasks. Given the development during the past decades, the time is now ripe for empirical evidence to catch up with theoretical predictions concerning the consequences of plasticity. This is challenging, because, as argued below, to empirically evaluate the predicted consequences (and other issues) of plasticity requires methods allowing for quantifying and comparing 'single trait' as well as 'whole organism' plasticity. In addition, I claim that rigorous testing of predictions requires that we can experimentally manipulate the levels of or the capacity for plasticity.

\section{WHAT IS PLASTICITY?}

As pointed out by others, the concept of phenotypic plasticity is deceptively simple, and it has been defined in numerous ways by different authors (see, for instance, Box 1 in Whitman and Agrawal, 2009). One apparent form of intraindividual plasticity, common to most if not all multicellular organisms, is the pronounced phenotypic differentiation among cells found in different organs and types of tissue (Sánchez Alvarado and Yamanaka, 2014). Cells that comprise blood, bone, nerve and muscle within a single individual are phenotypically extremely different, despite that they share an identical (at least close to) set of genes and alleles. Such induced differentiation has previously been discussed from a plasticity perspective within the domains of ecology and evolutionary biology (see, for example, Sarà, 1996; Newman and Müller, 2000; Schlichting, 2003).

Plasticity is oftentimes defined as the ability of a single genotype to exhibit a range of different phenotypes in response to variation in the environment. The term phenotypic plasticity is used in studies that concern and report on phenomena that are in fact rather different, 
and alternative terms are sometimes used for one and the same phenomenon (Piersma and Drent, 2003; Whitman and Agrawal, 2009; Stamps and Groothuis, 2010; Brommer, 2013; Snell-Rood, 2013). The same or similar concepts are sometimes also used for different phenotypic dimensions, such as morphology, physiology, life history and behaviour, and for situations when the phenotype changes in response to variation in either the external or the internal environment. On one hand, the tendency to apply the same concept to different types of context-dependent phenotypic variation can be justified on the grounds that science may benefit from unifying, common theoretical frameworks. On the other hand, it is sometimes justified to use different concepts. Given the rich terminology (Table 1), however, authors should take great care to clarify how they interpret and use the plasticity concept and related terms to avoid misunderstandings, because people vary in what they think plasticity is and what it is not.

\section{Active versus passive plasticity}

One distinction worth mentioning in this context is that between active and passive plasticity (Scheiner, 2006; Kurashige and Callahan, 2007; Whitman and Agrawal, 2009), because there might be differences in the way that these two aspects of plasticity affect the ecological success of individuals and populations. Active plasticity is used for predominantly anticipatory, and often highly integrated, phenotypic changes in response to some environmental cue or signal, and reflect modifications of developmental pathways and regulatory genes. In active plasticity, such as in the case of wing-length polymorphism in some species of insects (Roff and Fairbairn, 2007; Schwander and Leimar, 2011), the magnitude of the induced phenotypic response(s) is not necessarily correlated with the strength of the environmental signal (Scheiner, 2006). However, graded responses are also relatively common for active plasticity. For example, the magnitude of inducible defences in prey may increase as the number of predators, or the amount of cue produced by predators, increases. Passive plasticity, on the other hand, may stem from direct environmental influences on chemical, physiological and developmental processes, and is generally not considered anticipatory, but a mere consequence of the environment, such as stunted growth owing to low resource levels. In the case of passive plasticity, when the environment acts directly on the expression of the trait, phenotypic changes are often proportional to environmental differences (Scheiner, 2006). Active phenotypic plasticities are often (unlike 'truly' passive plasticities) considered adaptive, but the distinction between the two is not always clearcut (Scheiner, 2006; Whitman and Agrawal, 2009).

\section{Developmental plasticity versus phenotypic flexibility}

The term 'developmental plasticity' is used by many researchers who focus on irreversible phenotypic variation in traits of individuals (or genotypes) that result from environmentally induced modifications of development and growth. Some traits remain largely fixed after maturity, such as structural body size in some unitary organisms, the number of vertebrae in limbed vertebrates and various morphological defence structures induced by the presence of predators. Reaction norms (or norms of reaction) that describe the association linking the phenotypic expression of genotypes to an environmental gradient, and genotype by environment interactions that refer to the differential phenotypic responses of alternative genotypes, are key components in this field (Stearns, 1989; Roff, 1997; Lynch and Walsh, 1998; Pigliucci, 2001; West-Eberhard, 2003). Developmental plasticity is not restricted to traits that display quantitative variation, and it can result in discrete phenotypic classes that represent developmental threshold responses regulated by complex mechanisms including polygenic effects and shifts induced by continuously distributed underlying cues (Stearns, 1989). A candidate example is wing polymorphism, the co-occurrence of flight-capable individuals that possess fully developed functional wings and flightless individuals with only partially developed or no wings that in some insect species seems to be regulated by juvenile hormone titres (Zera, 2003; Roff and Fairbairn, 2007; Schwander and Leimar, 2011). Plasticity can also involve mechanisms that operate between or across generations. In quantitative genetics and evolutionary biology, when the environment responsible for the induced phenotypic response consist of the (female) parent, the developmental plasticity is usually referred to as maternal effects (Roff, 1997; Mousseau and Fox, 1998) or cross generational plasticity.

The term 'phenotypic flexibility' seems to be used primarily by researchers who focus on intraindividual plasticity, that is, reversible changes within individuals of labile, context-dependent physiological, morphological and life-history traits (Piersma and Drent, 2003). Examples include metabolic and endocrine switches, shifts in the sizes of body parts and organ systems in relation to reproductive condition and metabolic demand, changes in colour patterns in relation to seasonality, changes within individuals among years in the timing of reproductive activities in response to weather conditions and changes in number of eggs or offspring between sequential reproductive events. Phenotypic flexibility is also applied to changes in behavioural traits (Dingemanse et al., 2010; Stamps and Groothuis, 2010; Tuomainen and Candolin, 2011), for instance, as a result of learning and experience or adjustments depending on the presence or absence of predators, and encompasses both developmental behavioural plasticity and activational plasticity (for a thorough discussion see Snell-Rood, 2013). Despite the flexible nature of many behavioural traits, there is also a tendency of individuals to display consistency through time. In the behavioural plasticity literature, there is a growing interest in the causes and consequences of such consistency within individuals and repeatable variation among individuals, often referred to as animal personalities or 'behavioural syndromes', for instance in the form of shyness versus boldness in different types of behaviour and across environmental contexts (Stamps and Groothuis, 2010; Brommer, 2013; Araya-Ajoy and Dingemanse, 2014).

\section{Is plasticity distinct from genetic polymorphism?}

There are still articles published (including some of my own) that contain phrasings and arguments along the lines of genetics versus plasticity that might suggest that the authors do not consider plasticity a property of the genotype. There are probably examples of intraindividual reversible phenotypic flexibility and behavioural plasticity that are only loosely connected to genetics. Plasticity is not equivalent to genetic polymorphism, and their evolutionary dynamics can differ considerably (Day and Bonduriansky, 2011; Frank, 2011; Saito et al., 2013). However, irreversible developmental plasticity is not fundamentally different from genetic determinism, except for the environment dependence of the expression of the phenotypic state, and they are best interpreted and analysed within a common, quantitative genetics, framework (Agrawal, 2001; Pigliucci, 2001; West-Eberhard, 2003; Jablonka and Lamb, 2006; Leimar et al., 2006; Day and Bonduriansky, 2011; Wennersten and Forsman, 2012). Most phenotypic traits display a certain amount of plasticity, and plasticity includes genetic components (Stearns, 1989; Pigliucci, 1996, 2001; Pigliucci and Preston, 2004). Indeed, the existence of crossing norms of reaction, such that different genotypes display different phenotypic responses to environmental change, reflects an underlying genetic 
polymorphism (Stearns, 1989; Pigliucci, 2001; West-Eberhard, 2003). This means that population-level consequences of plasticity predicted by theory are largely mediated by the same mechanisms as are those of genetic polymorphisms (for exceptions see Wennersten and Forsman, 2012).

\section{WHAT IS A TRAIT?}

Not all types of traits can be straightforwardly assigned to either of the above categories of plasticity. For instance, the size and shape of antlers in mammals of the family Cervidae, such as deer, is an example of irreversible developmental plasticity, but new antlers are grown each year, and the antlers of an individual can change in both size and shape among years. Similarly, the size and colouration of feathers in birds remain largely fixed after feathers are fully grown, but can change within individuals between moults. This raises the question of whether the antlers of a deer, the plumage of a bird or aspects of behavioural and life-history characters as expressed on different occasions by the same individual should be considered as one or as different traits. One way to address such intraindividual plasticity is to quantify, compare and model individual reaction norms based on longitudinal data (Nussey et al., 2007; Araya-Ajoy and Dingemanse, 2014). In modular organisms, phenotypic responses to environmental cues can be differentiated and restricted to certain body compartments, such as size and shape of leaves subjected to sun-exposed versus shaded environments (Pigliucci et al., 1999, 2003), and aerial versus aquatic leaves in some plants (Bruni et al., 1996; Sultan, 2003). Interestingly, this enables the individual to be a generalist, and may be of particular importance for sessile organisms that cannot choose or move to a more suitable environment.

As another case in point, consider the plethora of factors that influence growth rate and body size in snakes (Figure 2). Snakes have indeterminate growth, meaning that they continue to grow more or less throughout life, although at a rate that decreases with increasing body size and age, and with males or females growing larger adult body sizes depending on species (Shine, 1991). Their growth rates are flexible and influenced by the internal environment, including genetic makeup and number of vertebrae (Lindell et al., 1993; Shine, 2000), as well as by the external environment, for instance in the form of prey availability (Forsman and Lindell, 1996; Lindell and Forsman, 1996; Forsman, 1996b). Unlike vertebrate taxa with limbs, snakes display considerable variation in the number of vertebrae, both among species and among individuals within populations (Arnold, 1988; Lindell et al., 1993; Lindell, 1994). Vertebral number reflects the combined effects of heritable (additive) genetic variation and developmental plasticity in response to temperature conditions during early embryonic development, and unlike growth rate and body size it does not change during the rest of the life of the individual (Arnold, 1988; Lindell et al., 1993; Shine, 2000 and references therein). Because snakes are gape-limited predators that swallow their prey in one piece, their foraging success depends on body size (Forsman and Lindell, 1993; Forsman, 1996a).

Through its effect on locomotor capacity and speed (Arnold and Bennett, 1988), there is also potential for vertebral number to indirectly influence foraging success and growth rate. Snakes are ectothermic, and their growth rates depend strongly on body temperature, as do virtually all aspects of their physiology and behaviour, including foraging activities (Huey and Kingsolver, 1989). Body temperature of snakes is in turn influenced by behavioural thermoregulation mediated via shifts in bodily postures and shuttling between microhabitats; examples of reversible phenotypic flexibility and behavioural plasticity (Stevenson, 1985b; Forsman, 1995). Body temperature of snakes and other ectothermic organisms is also influenced by external weather conditions, and by body colouration and body size, all of which affect the rate at which solar radiation is converted into body heat as well as equilibrium body temperature (Stevenson, 1985a; Forsman, 1995; Forsman et al., 2002; Ahnesjö and Forsman, 2006; Karpestam et al., 2012). This example illustrates that to apply the plasticity concept(s) to single 'traits' (for example, body size) as a means to increase our understanding of organismal design and differentiation among individuals and populations can be a daunting endeavour (Figure 2).

FROM SINGLE TRAITS TO A WHOLE ORGANISM PERSPECTIVE It can be argued that 'traits' do not really exist, except as mental constructions to aid communication (Fristrup, 2001). Indeed, individuals are developmentally, functionally and phenotypically integrated complex units (Olson and Miller, 1958; Schlichting and Pigliucci, 1998; Pigliucci and Preston, 2004; Valladares et al., 2007; Piersma and van Gils, 2011). Within an individual, homeostasis, canalization or the absence of plasticity (or a flat reaction norm for a genotype) in a focal

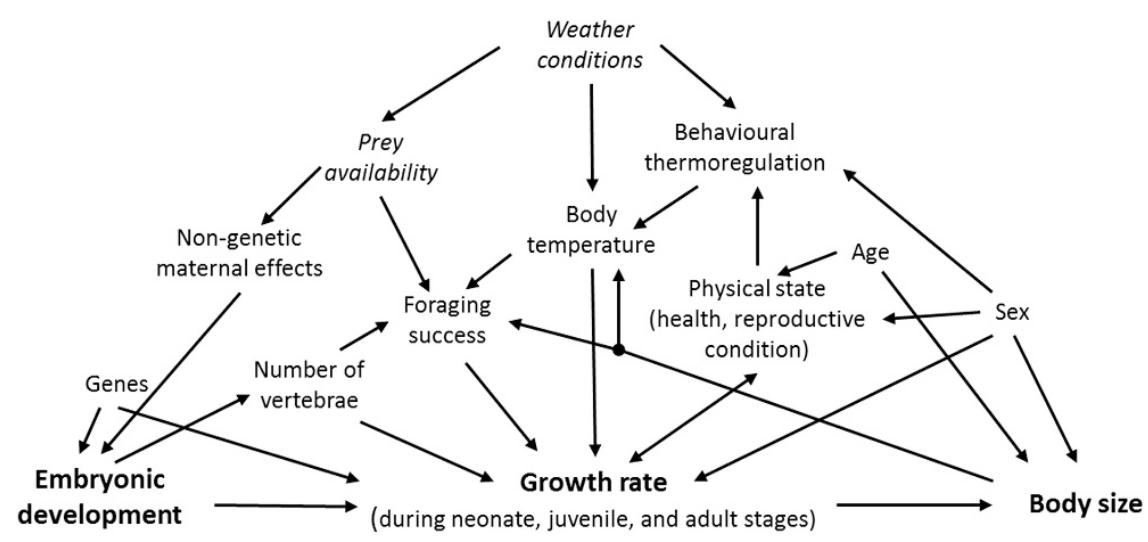

Figure 2 Interdependence among phenotypic dimensions, plasticity, flexibility and environmental influences jointly affect body size in snakes. Schematic representation of the many ways by which different phenotypic dimensions interact and are influenced by internal and external environmental factors and how they may jointly contribute to within- and among-individual variation in growth rate and body size of snakes. This example illustrates that individuals are complex integrated units that cannot be decomposed into a suite of independent 'traits', and that variation in a given phenotypic dimension can be influenced by combinations of both genes, irreversible developmental plasticity and by reversible phenotypic flexibility in response to changes along different environmental factors. See text for details. 
trait must reflect some sort of buffering mechanism and plasticity in some other trait(s) (Pigliucci and Preston, 2004; Whitman and Agrawal, 2009). Such buffering can be mediated by a flexibility of physiological processes, developmental pathways and by behavioural adjustments (Figure 2). Although developmental plasticity and phenotypic flexibility can enable individuals to change their phenotype according to the environment, mobile organisms may also have the opportunity to change their environment according to phenotype by means of matching habitat choice (Edelaar et al., 2008; Karpestam et al., 2012). This raises the questions of whether it is meaningful and possible from a whole organism perspective to classify individuals, populations or species as being either plastic or non-plastic

If one takes into consideration the entire integrated suite of physiological, behavioural, morphological and life-history phenotypic dimensions, it can be argued that there is no such thing as a nonplastic individual, because lack of or low levels of plasticity in one trait is generally compensated for or made possible by higher plasticity or flexibility along some other phenotypic dimension(s). This realization is important when it comes to making comparisons, evaluating hypotheses and testing predictions pertaining to the fitness consequences of plasticity for individuals and populations. To avoid misunderstandings, any inferences and conclusions with regard to causes and consequences of plasticity should be restricted to the particular phenotypic dimension(s), aspects of plasticity and environmental cues(s) that have been investigated, and not extended to general statements regarding plasticity.

\section{COMPARING LEVELS OF PLASTICITY AMONG INDIVIDUALS, POPULATIONS AND SPECIES}

Multicellular organisms are complex combinations of phenotypic properties that display more or less plasticity (Bradshaw, 1965; Sultan, 2000; Pigliucci, 2001; Schlichting, 2003; West-Eberhard, 2003; Pigliucci and Preston, 2004; Araya-Ajoy and Dingemanse, 2014). One should therefore not expect plasticity to be correlated across phenotypic dimensions or environments. A given genotype may show a plastic response (a non-flat reaction norm) of one or some particular dimension(s) of the phenotype in response to a certain environmental factor, whereas other phenotypic dimensions are not affected by the same environmental factor. Similarly, a given genotype or phenotypic dimension may display plasticity in response to changes along the gradient of one environmental factor, whereas changes along other environmental gradients do not affect the expression of the same genotype nor influence its associated phenotypic variability (Valladares et al., 2007). Conclusions regarding the consequences of plasticity responses and gene by environment interactions therefore depend on the choice of trait(s) as well as on the choice of environmental covariate(s) (Valladares et al., 2007; Brommer, 2013). Assessments of plasticity are typically focussed on traits with established or a priori functional significance and how they respond to changes along some ecologically important environmental gradient. Consequently, there is a risk with a reductionist approach that we might arrive at a biased view of, and perhaps even overestimate, the importance of plasticity. To improve our understanding of the evolutionary dynamics of plasticity and its consequences, it is necessary to also identify those environmental factors that do not elicit plastic responses, as well as the ecological settings under which plasticity is less beneficial.

A plastic response in a given phenotypic dimension can be demonstrated experimentally. However, to reliably classify genotypes, populations or species as being non-plastic is complicated because it is impossible to determine with certainty that observable phenotypic variation among individuals is not influenced at all by responses to any environmental cues. This is because the population or species under investigation may potentially show plasticity in response to environmental cue(s) that have not yet been investigated, or have a restricted window of sensitivity not yet tested (Pigliucci, 2001; West-Eberhard, 2003). Furthermore, the outcome of plasticity experiments conducted under laboratory conditions may not accurately reflect the role of plasticity under different and usually more complex and challenging circumstances in the wild (Nussey et al., 2007; Valladares et al., 2007; Fox and Reed, 2010). It may not be possible to reliably compare and rank individuals, populations or species with regard to some overall level of or capacity for whole organism plasticity. However, comparisons can be done at the level of specific phenotypic dimensions and with regard to how they respond to changes along the gradient of one or a few specified environmental factors. In lieu of estimates of whole organism plasticity, multiple phenotypic dimensions can be examined, modelled and compared simultaneously by either using multivariate statistical analysis or using composite measures of plasticity based on averages across traits or dimension reducing techniques (such as principal component analysis) as a means to inform about differences in the nature and magnitude of plasticity among individuals, populations and species (see, for example, Carroll and Corneli, 1995; Pigliucci et al., 1999; Pollard et al., 2001; Nussey et al., 2007; Chun, 2011; Davidson et al., 2011; Araya-Ajoy and Dingemanse, 2014).

\section{HOW CAN HYPOTHESES AND PREDICTIONS REGARDING CONSEQUENCES OF PLASTICITY BE TESTED?}

To put the predictions about plasticity to empirical tests, we must be able to quantify, compare and ultimately manipulate the level of and capacity to express plasticity. This is true not only for the proposed population-level consequences (Wennersten and Forsman, 2012); the need to quantify, compare and manipulate plasticity also applies if we want to investigate, for example, whether plasticity influences fitness, whether it is adaptive and whether it is costly (Tonsor et al., 2013).

\section{Associations based on observational data may be informative but} do not reveal causation

Indications of costs or benefits of plasticity might be gained from comparisons of performance of different genotypes or between populations subjected to different environmental conditions (Wund, 2012). Similarly, associations of variation in single- or multivariate trait plasticity with changes along environmental gradients can inform about which factors and conditions might promote the evolution of induced phenotypic variation. It is also possible to test for associations of plasticity with fitness in the wild, but this requires tedious analyses, and interpretation of results is not straight forward (Nussey et al., 2007; Brommer, 2013). For instance, Araya-Ajoy and Dingemanse (2014) proposed an analytical approach that allows for studying (co) variation in labile behavioural character complexes both within and among individuals using multivariate mixed-effects models. Their approach resembles to some extent the one put forward by Nussey et al. (2007) for quantifying, comparing and partitioning variation among individuals and populations in reaction norms of labile lifehistory traits based on longitudinal data from natural populations. Comparisons among populations that inhabit different environments may help identify potential drivers of plasticity evolution and suggest how plasticity can contribute to evolutionary differentiation within species (see, for example, Carroll and Corneli, 1995; Pollard et al., 2001; Nunes et al., 2013). On a larger scale, phylogeny-based comparative analyses can be used to infer about the role of plasticity for evolutionary diversification among species and for speciation (see, for example, Colbourne et al., 1997; Thaler and Karban, 1997; 
Pigliucci et al., 1999; Price et al., 2003; Pfennig et al., 2010; Schwander and Leimar, 2011). However, with a whole organism perspective on plasticity (that is, acknowledging that homeostasis in one particular phenotypic dimension is mediated by plasticity or flexibility in other dimension(s)), it is difficult to envisage how to perform such comparisons and demonstrate such associations - let alone to establish causation.

\section{How to manipulate plasticity in order to establish causation?}

Studies based on observational approaches can offer important insights, generate hypothesis and testable predictions and uncover patterns and associations in accordance or disagreement with predictions. However, demonstrating causal relationships and mechanisms linking either variation in the capacity for plasticity itself or plasticityinduced phenotypic variation to aspects of individual or population fitness is complicated because it requires experimental manipulation, replication and controlled comparisons (Forsman, 2014).

To experimentally manipulate the level of or capacity for plasticity seems very challenging, at least if one aims to test for effects of the capacity to express plasticity or flexibility (independent of genetic variation), and if one is concerned with whole organism rather than single trait plasticity. Artificial selection or genetic engineering might be used to increase or reduce levels of plasticity-induced phenotypic variation (Krebs and Feder, 1998; Feder, 1999). However, interpretation of such manipulation studies is not straight forward, because of the potential for pleiotropy and epistasis (Lynch and Walsh, 1998), and because changes in plasticity of the focal trait may be accompanied by increased or decreased plasticity in buffering traits. Phenotypic engineering based on hormonal treatments offers another possibility to experimentally manipulate the phenotypic expression of plastic and flexible traits (Sinervo and Huey, 1990; Ketterson et al., 1996), but again interpretation of results is complicated because hormones can have a multitude of cascading and interacting effects on organismal functioning. To test for effects and consequences of plasticity based on comparisons between experimental treatments that consist of unmanipulated individuals with naturally high versus low plasticity is equally problematic, because differences in plasticity between treatments would not be independent of genetic variation. Chevin et al. (2013) provide a discussion and offer suggestions on the related issue of experimental evolution of plasticity.

\section{POPULATION-LEVEL CONSEQUENCES OF PLASTICITY REVISITED}

In view of what has been said above, is there any rigorous evidence in support of the predictions (Table 1 in Wennersten and Forsman, 2012 and references therein) that plasticity should promote the ecological and evolutionary success of populations and species? It is important in this context to distinguish between effects mediated by variation in the capacity to express plasticity and flexibility versus consequences associated with a higher (or lower) level of interindividual phenotypic variation resulting from plasticity.

The buffering effect of plasticity-induced phenotypic variation against environmental change should apply primarily to labile or flexible traits, and to fine-grained environments where the changes are predictable (Wennersten and Forsman, 2012). Recent reviews and discussions of behavioural flexibility are largely in accordance with this prediction (Tuomainen and Candolin, 2011; Snell-Rood, 2013), but the evidence is based almost exclusively on observational data and theoretical modelling. For instance, Vedder et al. (2013) report on a quantitative assessment of the importance of plasticity in adaptation to climate change based on a long-term population study of wild great tits (Parus major) and conclude that the most important way that birds can cope with climate change is their evolved ability to adjust their behaviour and seasonal timing of reproductive activities depending on spring temperature and abundance of a critical food resource, with extinction risk being 500 times higher in the absence of plasticity. Modelling approaches suggest that developmental variability and learning can enhance nonheritable phenotypic variation and, by smoothening of multipeaked landscapes that relate genotypes to fitness, this can in principle affect both the course and the rate of evolution to a fitness peak, and it can do so in changing as well as in 'fixed' environments (see, for example, Ghalambor et al., 2007; Frank, 2011; Saito et al., 2013). However, although plasticity can accelerate evolutionary rate, there are indications that it may decrease the average fitness (Saito et al., 2013).

In the case of developmental plasticity, it has been stated that it may enhance establishment success, promote invasiveness, decrease vulnerability to environmental change and reduce extinction risk because it allows for individuals (or their progeny) to develop phenotypes that are well suited to novel conditions without first undergoing local genetic adaptation through natural selection (see, for example, Sultan, 2000; Pfennig et al., 2010). Forsman (2014) reported on the results of a meta-analysis of experimental manipulation studies of plants and animals, demonstrating that greater genetic and phenotypic variation among individuals included in founder groups contribute to increased establishment success, but that study did not aim to evaluate the effects of plasticity on establishment. The reason for this exclusion was that, to my knowledge, there are as yet no studies that have experimentally manipulated the capacity for plasticity to investigate whether and how this might affect establishment and population persistence. In contrast, Davidson et al. (2011) reported on the results of a meta-analysis that indicate that invasive plant species display higher phenotypic plasticity compared with co-occurring, closely related, noninvasive species. They used for their comparisons both separate traits and a composite measure of species plasticity based on means across different traits. On a larger scale, it has been suggested that plasticity promotes evolutionary diversification and speciation (Price et al., 2003; West-Eberhard, 2003; Pfennig and McGee, 2010; Pfennig et al., 2010; Pigliucci, 2010), but again experimental evidence seems to be lacking.

The role of plasticity in some contexts should be contingent upon the spatial and temporal scales of environmental heterogeneity and change, relative to the dispersal capacity and generation time of the organisms (Levins, 1968; Frank and Slatkin, 1990; Saito et al., 2013). It is conceivable that if there is a substantial time lag between exposure to the environmental cue and the expression of the induced response, selection may drive the population to extinction before the appropriate phenotype has been realized (Wennersten and Forsman, 2012). Costs associated with such time lags are likely to be manifest most strongly for irreversible developmental plasticity when the induced change is not realized until the next generation. This hypothesis could potentially be evaluated experimentally by first using a split-brood design, exposing half of the siblings to a range of environmental conditions to increase the level of interindividual phenotypic variation, and the other half to homogeneous conditions, and then comparing the establishment success, population dynamics and persistence of phenotypically highly variable versus less variable founder groups. This would not answer the question of whether the capacity for plasticity buffers against environmental change, but would indicate whether standing phenotypic variation resulting from plasticity promotes ecological success of populations. 
Whether or not plasticity is beneficial for fitness of individuals and viability of populations is also contingent on how reliably the cues predict future environmental conditions and selective regimes. For instance, Reed et al. (2010) presented results of a stochastic individualbased model and found that demographic consequences of plasticity depend in a complex, nonadditive way on the reliability of environmental cues and the magnitude of environmental fluctuations. When environmental variation was highly unpredictable, strong plasticity increased rather than decreased extinction risk (Reed et al., 2010). See also Chevin et al. (2013) for a discussion of this issue.

Taken together, it seems that plasticity may either promote or impair ecological success of populations, depending on environmental conditions. However, firm evidence to this effect based on experimental manipulation approaches is lacking. Solving the problem of how to manipulate the capacity for developmental plasticity and flexibility is a daunting task, but it is crucial for continued scientific progress and to further our understanding of the many ways by which environmental heterogeneity and change may influence the evolution of genetic and phenotypic diversity at different levels of biological organization on one hand, and to investigate how plasticity affects the ecological success on the other hand.

\section{CONCLUSIONS AND FUTURE DIRECTIONS}

- Plasticity research has grown tremendously, from $<10$ papers published per year before 1983 to nearly 1300 papers in 2013. There has been an increased interest in the questions of how interindividual variation and plasticity influences the performance and ecological success of individuals, populations and species.

- Our understanding of causes and consequences of plasticity and its role in generating and maintaining phenotypic complexity and diversity is hampered by imprecise definitions, hypothesis and predictions, and a tendency to consider observational case studies as evidence in support of predictions and confirmation of theory. Authors should take great care to clarify how they interpret and use plasticity and related terms.

- Within an individual, homeostasis, canalization or the absence of plasticity (a flat reaction norm) in a focal trait must be compensated for by higher plasticity or flexibility along some other phenotypic dimension(s); it can thus be argued that there is no such thing as a non-plastic individual.

- Inferences and conclusions with regard to causes and consequences of plasticity should be restricted to the particular phenotypic dimension(s), aspects of plasticity and environmental cues(s) that have been investigated, and not extended to general statements regarding plasticity as such, unless a whole organism approach is used.

- The consequences of plasticity may differ depending on whether one is concerned with effects mediated by variation among individuals in the capacity to express plasticity and flexibility, or with effects driven by greater interindividual phenotypic variation resulting from induced responses (in which case there need be no genetic variation in plasticity).

- To put predictions to empirical tests, we must be able to quantify and compare the nature and magnitude of plasticity among individuals, populations and species. In the wait of estimates of whole organism plasticity, multiple phenotypic dimensions can be examined, modelled and compared using multivariate statistical analysis or by using composite measures of plasticity based on averages across traits or dimension reducing techniques.
- To improve our understanding of the evolutionary dynamics of plasticity and its consequences, and to avoid overestimating its importance, it is necessary to identify also those phenotypic dimensions that are less likely to express plasticity or flexibility, those environmental factors that do not elicit plastic responses as well as the ecological settings under which plasticity is less beneficial.

- Past and current research, based on theoretical modelling and observational data, indicates that plasticity may either promote or impair ecological success of populations, depending on environmental conditions. However, firm evidence to this effect is lacking.

- To demonstrate causal relationships and mechanisms linking plasticity variation to aspects of individual or population fitness requires methods allowing for experimental manipulation, replication and controlled comparisons. It is also important to distinguish between effects mediated by variation in the capacity to express plasticity and flexibility, as opposed to effects mediated by a higher (or lower) level of interindividual phenotypic variation resulting from plasticity (in which case there need be no genetic variation in plasticity).

- Whether and how standing phenotypic variation resulting from plasticity influences aspects of ecological success of populations can be experimentally investigated by first using a split-brood design to increase and decrease interindividual phenotypic variation and then comparing for instance establishment success, population dynamics and persistence of phenotypically highly variable versus less variable groups.

- To experimentally demonstrate that interindividual genetic and phenotypic variation affects ecological success of populations and species is tedious, but feasible (Hughes et al., 2008; Wennersten and Forsman, 2012; Forsman, 2014). However, to disentangle any effects of whole organism plasticity from effects owing to genetic variation, and to rigorously investigate how variation in the capacity to express plasticity affects fitness of individuals and on the ecological success of populations and species, requires experimental approaches yet to be discovered. $\mathrm{S} /$ he who finds a solution to this problem will have a key to considerable future scientific advance. Let the quest for this Holy Grail begin!

\section{CONFLICT OF INTEREST}

The author declares no conflict of interest.

\section{ACKNOWLEDGEMENTS}

I thank the organizers, most especially Johan Hollander, and participants of the Symposium on 'Phenotypic Plasticity-Variation, Alteration and Speciation' held in Lund, November 2012, for inspiration. I am grateful to H Berggren, S Hylander, P Tibblin, J Tinnert, L Wennersten and to four anonymous reviewers for discussion and comments on the manuscript. This work was funded by The Swedish Research Council and Linnaeus University.

\footnotetext{
Agrawal AA (2001). Phenotypic plasticity in the interactions and evolution of species. Science 294: 321-326.

Ahnesjö J, Forsman A (2006). Differential habitat selection by pygmy grasshopper color morphs; interactive effects of temperature and predator avoidance. Evol Ecol 20: 235-257.

Araya-Ajoy YG, Dingemanse NJ (2014). Characterizing behavioural 'characters': an evolutionary framework. Proc $R$ Soc B 281: 20132645.

Arnold SJ, Bennett AF (1988). Behavioral variation in natural populations. V. Morphological correlates of locomotion in the garter snake Thamnophis radix. Biol J Linn Soc 34 $175-190$
} 
Arnold SJ. (1988). Proceedings of the Second Interntaional Conference on Quantitative Genetics. In: Weir BS, Goodman MM, Eisen EJ, Namkong G (eds). Sinauer Associates, pp 619-636.

Bolnick DI, Svanbäck R, Fordyce JA, Yang LH, Davis JM, Hulsey CD et al. (2003). The ecology of individuals: incidence and implications of individual specialization. Am Nat 161: 1-28.

Booth RE, Grime JP (2003). Effects of genetic impoverishment on plant community diversity. J Ecol 91: 721-730.

Bradshaw AD (1965). Evolutionary significance of phenotypic plasticity in plants. Adv Genet 13: 115-155.

Brommer JE (2013). Phenotypic plasticity of labile traits in the wild. Curr Zool 59: 485-505.

Bruni NC, Young JP, Dengler NG (1996). Leaf developmental plasticity of Ranunculus flabellaris in response to terrestrial and submerged environments. Can J Bot 74: 823-837.

Carroll SP, Corneli PS (1995). Divergence in male mating tactics between two populations of the soapberry bug: II. Genetic change and the evolution of a plastic reaction norm in a variable social environment. Behav Ecol 6: 46-56.

Chevin L-M, Gallet R, Gomulkiewicz R, Holt RD, Fellous S (2013). Phenotypic plasticity in evolutionary rescue experiments. Philos Trans R Soc B Biol Sci 368: 20120089.

Chevin L-M, Lande R, Mace GM (2010). Adaptation, plasticity and extinction in a changing environment: towards a predictive theory. PLoS Bio/ 8: e1000357.

Chun YJ (2011). Phenotypic plasticity of introduced versus native purple loosestrife: univariate and multivariate reaction norm approaches. Biol Invasions 13: 819-829.

Colbourne JK, Herbert PDN, Taylor DJ (1997). Evolutionary origins of phenotypic diversity in Daphnia. In: Givnish TJ, Systma KJ (eds). Molecular Evolution and Adaptive Radiation. Cambridge University Press: Cambridge, pp 163-188.

Davidson AM, Jennions M, Nicotra AB (2011). Do invasive species show higher phenotypic plasticity than native species and, if so, is it adaptive? A meta-analysis. Ecol Lett 14: 419-431.

Day T, Bonduriansky R (2011). A unified approach to the evolutionary consequences of genetic and nongenetic inheritance. Am Nat 178: E18-E36.

Dingemanse NJ, Kazem AJN, Réale D, Wright J (2010). Behavioural reaction norms: animal personality meets individual plasticity. Trends Ecol Evol 25: 81-89.

Edelaar P, Siepielski AM, Clobert J (2008). Matching habitat choice causes directed gene flow: a neglected dimension in evolution and ecology. Evolution 62: 2462-2472.

Feder ME (1999). Engineering candidate genes in studies of adaptation: the heat-shock protein Hsp70 in Drosophila melanogaster. Am Nat 154: S55-S66.

Forsman A (1995). Heating rates and body temperature variation in melanistic and zigzag Vipera berus: does colour make a difference? Ann Zool Fennici 32: 365-374.

Forsman A (1996a). Body size and net energy gain in gape-limited predators: a model. J Herpetol 30: 307-319.

Forsman A (1996b). An experimental test for food effects on head size allometry in juvenile snakes. Evolution 50: 2536-2542.

Forsman A (2014). Effects of genotypic and phenotypic variation on establishment are important for conservation, invasion and infection biology. Proc Natl Acad Sci USA 111: 302-307.

Forsman A, Ahnesjö J, Caesar S, Karlsson M (2008). A model of ecological and evolutionary consequences of color polymorphism. Ecology 89: 34-40.

Forsman A, Lindell E (1996). Resource dependent growth and body condition dynamics in juvenile snakes: an experiment. Oecologia 108: 669-675.

Forsman A, Lindell LE (1993). The advantage of a big head - swallowing performance in adders, Vipera berus. Funct Ecol 7: 183-189.

Forsman A, Ringblom K, Civantos E, Ahnesjö J (2002). Coevolution of color pattern and thermoregulatory behavior in polymorphic pygmy grasshoppers Tetrix undulata. Evolution 56: 349-360.

Fox CW, Reed DH (2010). Inbreeding depression increases with environmental stress: an experimental study and meta-analysis. Evolution 65: 246-258.

Frank SA (2011). Natural selection. II. Developmental variability and evolutionary rate. J Evol Biol 24: 2310-2320.

Frank SA, Slatkin M (1990). Evolution in a variable environment. Am Nat 136 244-260.

Frankham R, Ballou JD, Briscoe DA (2010). Introduction to Conservation Genetics, 2nd edn. Cambridge University Press: Cambridge, UK.

Fristrup KM (2001). A history of character concepts in evolutionary biology. In: Wagner GP (ed). The Character Concept in Evolutionary Biology. Academic Press: San Diego, pp 13-35.

Ghalambor CK, McKay JK, Carroll SP, Reznick DN (2007). Adaptive versus non-adaptive phenotypic plasticity and the potential for contemporary adaptation in new environments. Funct Ecol 21: 394-407.

Gray SM, McKinnon JS (2007). Linking color polymorphism maintenance and speciation. Trends Ecol Evol 22: 71-79.

Huey RB, Kingsolver JG (1989). Evolution of thermal sensitivity of ectotherm performance. Trends Ecol Evol 4: 131-135.

Hughes AR, Inouye BD, Johnson MTJ, Underwood N, Vellend M (2008). Ecological consequences of genetic diversity. Ecol Lett 11: 609-623.

Jablonka E, Lamb MJ (2006). Evolution in Four Dimensions. MIT Press: Cambridge, MA.

Karpestam E, Wennersten L, Forsman A (2012). Matching habitat choice by experimentally mismatched phenotypes. Evol Ecol 26: 893-907.

Kendall BE, Fox GA (2002). Demographic factors and genetic variation influence population persistence under environmental change. Conserv Biol 16: 109-116.
Ketterson ED, Nolan V Jr., Cawthorn MJ, Parker PG, Ziegenfus C (1996). Phenotypic engineering: using hormones to explore the mechanisms and functional bases of phenotypic variation in nature. Ibis 138: 70-86.

Krebs RA, Feder ME (1998). Experimental manipulation of the cost of thermal acclimation in Drosophila melanogaster. Biol J Linn Soc 63: 593-601.

Kurashige NS, Callahan HS (2007). Evolution of active and passive forms of plasticity: insights from artificially selected Arabidopsis. Evol Ecol Res 9: 1-11.

Leimar $O$ (2009). Environmental and genetic cues in the evolution of phenotypic plasticity. Evol Ecol 23: 125-135.

Leimar O, Hammerstein P, Van Dooren TJM (2006). A new perspective on developmental plasticity and the principles of adaptive morph determination. Am Nat 167: 367-376.

Levins R (1968). Evolution in Changing Environments. Princeton University Press: Princeton.

Lindell LE (1994). The evolution of vertebral number and body size in snakes. Funct Ecol 8: 708-719.

Lindell LE, Forsman A (1996). Density effects and snake predation: prey limitation and reduced growth rate of adders at high density of conspecifics. Can $J$ Zool 74: 1000-1007.

Lindell LE, Forsman A, Merilä J (1993). Variation in number of ventral scales in snakes effects on body size, growth-rate and survival in the adder, Vipera berus. $J$ Zool 230: 101-115.

Lynch M, Walsh B (1998). Genetics and Analysis of Quantitative Traits. Sinauer Associates, Inc.: Sunderland, MA.

Meyers LA, Bull JJ (2002). Fighting change with change: adaptive variation in an uncertain world. Trends Ecol Evol 17: 551-557.

Miner BG, Sultan SE, Morgan SG, Padilla DK, Relyea RA (2005). Ecological consequences of phenotypic plasticity. Trends Ecol Evol 20: 685-692.

Mousseau TA, Fox CW (1998). The adaptive significance of maternal effects. Trends Ecol Evol 13: 403-407

Naeem S, Bunker DE, Hector A, Loreau M, Perrings C (eds) (2009). Biodiversity, Ecosystem Functioning, and Human Wellbeing. An Ecological and Economic Perspective. Oxford University Press: Oxford, pp 356.

Newman SA, Müller GB (2000). Epigenetic mechanisms of character origination. J Exp Zool 288: 304-317.

Nunes AL, Orizaola G, Laurila A, Rebelo R (2013). Rapid evolution of constitutive and inducible defenses against an invasive predator. Ecology 95: 1520-1530.

Nussey DH, Wilson AJ, Brommer JE (2007). The evolutionary ecology of individual phenotypic plasticity in wild populations. J Evol Biol 20: 831-844.

Olson EC, Miller RL (1958). Morphological Integration. University of Chicago Press: Chicago

Pfennig DW, McGee M (2010). Resource polyphenism increases species richness: a test of the hypothesis. Philos Trans R Soc B Biol Sci 365: 577-591.

Pfennig DW, Wund MA, Snell-Rood EC, Cruickshank T, Schlichting CD, Moczek AP (2010). Phenotypic plasticity's impact on diversification and speciation. Trends Ecol Evol 25: $459-467$.

Piersma T, Drent J (2003). Phenotypic flexibility and the evolution of organismal design. Trends Ecol Evol 18: 228-233.

Piersma T, van Gils JA (2011). The Flexible Phenotype: A Body-Centred Integration of Ecology, Physiology, and Behaviour. Oxford University Press.

Pigliucci M (1996). How organisms respond to environmental changes: from phenotypes to molecules (and vice versa). Trends Ecol Evol 11: 168-173.

Pigliucci M (2001). Phenotypic Plasticity Beyond Nature and Nurture. The John Hopkins University Press: Baltimore.

Pigliucci M (2005). Evolution of phenotypic plasticity: where are we going now? Trends Ecol Evol 20: 481-486

Pigliucci M (2010). Phenotypic plasticity. In: Pigliucci M, Müller GB (eds). Evolution - The Extended Synthesis. Massachusetts Institute of Technology: Massachusetts, pp 355-378.

Pigliucci M, Cammell K, Schmitt J (1999). Evolution of phenotypic plasticity a comparative approach in the phylogenetic neighbourhood of Arabidopsis thaliana. J Evol Biol 12: 779-791.

Pigliucci M, Pollard H, Cruzan MB (2003). Comparative studies of evolutionary responses to light environments in Arabidopsis. Am Nat 161: 68-82.

Pigliucci M, Preston K (2004). Phenotypic Integration: Studying the Ecology and Evolution of Complex Phenotypes. Oxford University Press: Oxford.

Pollard H, Cruzan M, Pigliucci M (2001). Comparative studies of reaction norms in Arabidopsis. I. Evolution of response to daylength. Evol Ecol Res 3: $129-155$.

Price TD, Qvarnström A, Irwin DE (2003). The role of phenotypic plasticity in driving genetic evolution. Proc $R$ Soc B 270: 1433-1440.

Reed TE, Waples RS, Schindler DE, Hard JJ, Kinnison MT (2010). Phenotypic plasticity and population viability: the importance of environmental predictability. Proc $R$ Soc $B$ 277: 3391-3400.

Roff DA (1997). Evolutionary Quantitative Genetics. Chapman \& Hall: New York.

Roff DA, Fairbairn DJ (2007). The evolution and genetics of migration in insects. Bioscience 57: 155-164.

Saito N, Ishihara S, Kaneko K (2013). Baldwin effect under multipeaked fitness landscapes: phenotypic fluctuation accelerates evolutionary rate. Physical Review E 87: 052701.

Sarà M (1996). A "sensitive" cell system. Its role in a new evolutionary paradigm. Riv Biol 89: 139-156. 
Scheiner SM (2006). Genotype-environment interactions and evolution. In: Fox CW, Wolf CM (eds). Evolutionary Genetics: Concepts and Case Studies. Oxford University Press: New York, pp 326-338.

Scheiner SM, DeWitt TJ (2004). Future research directions. In: DeWitt TJ, Scheiner SM (eds). Phenotypic Plasticity: Funcitonal and Conceptual Approaches. Oxford University Press: New York, pp 201-207.

Schlichting CD (2003). Origins of differentiation via phenotypic plasticity. Evol Dev 5: 98-105.

Schlichting CD, Pigliucci M (1998). Phenotypic Evolution: A Reaction Norm Perspective. Sinauer Associates: Sunderland, MA, USA.

Schwander T, Leimar 0 (2011). Genes as leaders and followers in evolution. Trends Ecol Evol 26: 143-151.

Shine R (1991). Australian Snakes. A Natural History. Reed: Sydney.

Shine R (2000). Vertebral numbers in male and female snakes: the roles of natural, sexual and fecundity selection. J Evol Biol 13: 455-465.

Shuster SM (2010). Alternative mating strategies. In: Westneat DF, Fox CW (eds). Evolutionary Behavioral Ecology. Oxford Univeristy Press: New York, pp 434-450.

Sinervo B, Huey R (1990). Allometric engineering: an experimental test of the causes of interpopulational differences in performance. Science 248: 1106-1109.

Snell-Rood EC (2013). An overview of the evolutionary causes and consequences of behavioural plasticity. Anim Behav 85: 1004-1011.

Stamps J, Groothuis TGG (2010). The development of animal personality: relevance, concepts and perspectives. Biol Rev 85: 301-325.

Stearns SJ (1989). The evolutionary significance of phenotypic plasticity - phenotypic sources of variation among organisms can be described by developmental switches and reaction norms. Bioscience 39: 436-445.

Stevenson RD (1985a). Body size and limits to the daily range of body temperature in terrestrial ectotherms. Am Nat 125: 102-117.

Stevenson RD (1985b). The relative importance of behavioral and physiological adjustments controlling body temperature in terrestrial ectotherms. Am Nat 126 362-386.

Sultan SE (2000). Phenotypic plasticity for plant development, function and life history. Trends in Plant Science 5: 537-542.

Sultan SE (2003). Phenotypic plasticity in plants: a case study in ecological development. Evol Dev 5: 25-33.

Sultan SE (2004). Promising directions in plant phenotypic plasticity. Persp Plant Eco Evol Syst 6: 227-233.

Sánchez Alvarado A, Yamanaka S (2014). Rethinking differentiation: stem cells, regeneration, and plasticity. Cell 157: 110-119.

te Beest M, Le Roux JJ, Richardson DM, Brysting AK, Suda J, Kubešová M et al. (2011). The more the better? The role of polyploidy in facilitating plant invasions. Ann Bot 109 $19-45$.
Thaler JS, Karban R (1997). A phylogenetic reconstruction of constitutive and induced resistance in Gossypium. Am Nat 149: 1139-1146.

Tonsor SJ, Elnaccash TW, Scheiner SM (2013). Developmental instability is genetically correlated with phenotypic plasticity, constraining heritability, and fitness. Evolution 67: 2923-2935.

Tuomainen U, Candolin U (2011). Behavioural responses to human-induced environmental change. Biol Rev 86: 640-657.

Valladares F, Gianoli E, Gómez JM (2007). Ecological limits to plant phenotypic plasticity. New Phytologist 176: 749-763.

Vedder O, Bouwhuis S, Sheldon BC (2013). Quantitative assessment of the importance of phenotypic plasticity in adaptation to climate change in wild bird populations. PLOS Biol 11: e1001605.

Violle C, Enquist BJ, McGill BJ, Jiang L, Albert CH, Hulshof C et al. (2012). The return of the variance: intraspecific variability in community ecology. Trends Ecol Evol 27: 244-252.

Wennersten L, Forsman A (2012). Population-level consequences of polymorphism, plasticity and randomized phenotype switching: a review of predictions. Biol Rev 87: 756-767.

West-Eberhard MJ (2003). Developmental Plasticity and Evolution. Oxford University Press: Oxford.

Whitlock R, Grime JP, Booth R, Burke T (2007). The role of genotypic diversity in determining grassland community structure under constant environmental conditions. J Ecol 95: 895-907.

Whitman DW, Agrawal AA (2009). What is phenotypic plasticity and why is it important? In: Whitman DW, Ananthakrishnan TN (eds). Phenotypic Plasticity of Insects: Mechanisms and Consequences. Science Publishers: Enfield, pp 1-63.

Wund MA (2012). Assessing the impacts of phenotypic plasticity on evolution. Integr Comp Biol 52: 5-15.

Zera AJ (2003). The endocrine regulation of wing polymorphism in insects: state of the art, recent surprises, and future directions. Integr Comp Biol 43: 607-616.

(c) (i) (2) (2) This work is licensed under a Creative Commons cc) Attribution-NonCommercial-ShareAlike 3.0 Unported License. The images or other third party material in this article are included in the article's Creative Commons license, unless indicated otherwise in the credit line; if the material is not included under the Creative Commons license, users will need to obtain permission from the license holder to reproduce the material. To view a copy of this license, visit http://creativecommons.org/licenses/by-nc-sa/3.0/ 